\title{
STAT1 negatively regulates hepatocellular carcinoma cell proliferation
}

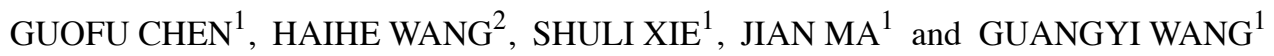 \\ ${ }^{1}$ Department of General Surgery, The First Hospital of Jilin University, Changchun, Jilin 130021; \\ ${ }^{2}$ Department of Pathogenobiology, Daqing Branch of Harbin Medical University, Daqing 163319, P.R. China
}

Received January 3, 2013; Accepted March 19, 2013

DOI: $10.3892 /$ or.2013.2398

\begin{abstract}
Signal transducer and activator of transcription 1 (STAT1) regulates cell proliferation and survival. The present study aimed to investigate the role of STAT1 in the development and progression of human hepatocellular carcinoma (HCC). The levels of STAT1 expression in $36 \mathrm{HCC}$ and 12 non-HCC liver tissues were examined by immunohistochemistry. The effect of STAT1 overexpression or silencing on the proliferation and apoptosis of HCC cells was determined by MTT and flow cytometric assays. The effect of STAT1 overexpression or silencing on the levels of p53 and cyclin E expression was determined by quantitative PCR and western blot assays. The level of STAT1 expression in the HCC tissues was significantly lower compared to the level in the non-HCC liver tissues and was negatively associated with the histological grade of HCC and serum HBsAg, anti-HCV and $\alpha$-fetoprotein positivity in HCC patients. Induction of STAT1 overexpression significantly inhibited HepG2 cell proliferation and enhanced HCC cell apoptosis, accompanied by upregulation of p53 expression and STAT1 phosphorylation, but a reduction in cyclin E expression in HepG2 cells. In contrast, knockdown of STAT1 by introduction of STAT1-specific siRNA promoted HepG2 cell proliferation, but inhibited HCC cell apoptosis, accompanied by significant downregulation of p53 expression, but enhancement of cyclin $\mathrm{E}$ expression in vitro. Our data suggest that STAT1 may inhibit HCC growth by regulating p53-related cell cycling and apoptosis.
\end{abstract}

\section{Introduction}

Hepatocellular carcinoma (HCC) is one of the most common malignant tumors worldwide (1). Its incidence is increasing due to a high prevalence of chronic liver diseases. Emerging lines of evidence indicate that aberrant activation of several signaling

Correspondence to: Professor Guangyi Wang, Department of General Surgery, The First Hospital of Jilin University, 71 Xin Min Street, Changchun, Jilin 130021, P.R. China

E-mail: guofuch163@163.com

Key words: STAT1, hepatocellular carcinoma, HepG2, proliferation, apoptosis cascades, including the Janus kinase/signal transducer and activator of transcription (Jak/STAT), epidermal growth factor receptor (EGFR), Ras/extracellular signal-regulated kinase (ERK), and phosphoinositol 3-kinase (PI3K)/mammalian target of rapamycin (mTOR) pathways, contributes to the pathogenesis of HCC (2). In addition, other factors, such as hepatocyte growth factor (HGF), mesenchymal-epithelial transition (MET) factors of Wnt, and Hedgehog, and apoptotic signaling, regulate the development and progression of HCC (2). However, how these signaling events regulate the development and progression of HCC are not fully understood. Hence, further clarification of individual signaling events in the development and progression of HCC will be of great significance.

The signal transducer and activator of transcription 1 (STAT1) functions as an important upstream regulator of interferon (IFN) signaling $(3,4)$. In many circumstances, STAT1 has both pro-apoptotic and anti-proliferative activities in tumor cells (5). STAT1-deficient mice are more prone to tumor development than animals with wild-type $(6,7)$. Indeed, chronic ethanol exposure induces oxidative stress and hepatocyte damage by inhibiting IFN- $\gamma$-mediated STAT1 activation (8). In addition, STAT1 is crucial for the control of hepatitis $\mathrm{C}$ virus (HCV) replication although the $\mathrm{HCV}$ core protein can selectively degrade STAT1, and subsequently subvert Jak-STAT kinase (9). It is possible that STAT1 may negatively regulate the growth of $\operatorname{HCC}(10,11)$. However, how STAT1 regulates the growth of HCC has not been clarified.

In the present study, we compared the levels of STAT1 expression in $36 \mathrm{HCC}$ and 12 non-HCC liver tissues as well as HCC cell lines, and analyzed their clinical relevance in HCC patients. Furthermore, we tested the impact of induction or knockdown of STAT1 expression on the proliferation and apoptosis of HepG2 cells and on the levels of p53, cyclin E, and STAT1 phosphorylation in HepG2 cells in vitro. Our data suggest that STAT1 may be a negative regulator of the development and progression of human HCC by regulating p53-related cell cycling and apoptosis.

\section{Materials and methods}

Patients and specimens. A total of 36 patients with HCC were recruited at the First Affiliated Hospital of Jilin University from April 2009 to April 2012. There were 21 male patients 
Table I. Sequences of siRNAs.

\begin{tabular}{lll}
\hline Gene & & \multicolumn{1}{c}{ Sequences } \\
\hline siRNA1:STAT1_homo-575 & Sense & 5'-GCUGGAUGAUCAAUAUAGUTT-3' \\
siRNA2:STAT1_homo-647 & Antisense: & 5'-ACUAUAUUGAUCAUCCAGCTT-3' \\
& Sense: & 5'-GCGUAAUCUUCAGGAUAAUTT-3' \\
siRNA3:STAT1_homo-1601 & Antisense: & 5'-AUUAUCCUGAAGAUUACGCTT-3' $^{\prime}$ \\
siRNA negative control & Sense: & 5'-GCACCUGCAAUUGAAAGAATT-3' \\
& Antisense: & 5'-UUCUUUCAAUUGCAGGUGCTT-3' \\
& Sense: & 5'-UUCUCCGAACGUGUCACGUTT-3' \\
& Antisense: & 5'-ACGUGACACGUUCGGAGAATT-3'
\end{tabular}

and 15 females with a median age of 50 and ranging from 34 to 73 years. Individual patients with $\mathrm{HCC}$ were diagnosed by radiological imaging and histological evidence of liver biopsy, and they underwent surgical removal of HCC. In addition, 12 gender and age-matched non-HCC patients were recruited for surgical resection of hepatic hemangioma, focal nodular hyperplasia, hepatic angiomyolipoma, or angioleiomyoma, and their non-HCC liver specimens were sampled. No patient received any chemotherapy or radiotherapy before sample collection. Individuals with acute inflammatory liver diseases were excluded. Written informed consent was obtained from each patient, and the experimental protocols were approved by the Ethics Committee of the First Affiliated Hospital of Jilin University.

Laboratory tests. Individual HCC tissue sections were stained with hematoxylin and eosin. The pathological degrees of HCC were graded into high, moderate or poor differentiation in a blinded manner. The concentrations of serum $\alpha$-fetoprotein (AFP), hepatitis B surface antigen (HBsAg), and anti-hepatitis $\mathrm{C}$ virus (anti-HCV) were measured by routine methods.

Immunohistochemical analysis. The levels of STAT1 expression in the specimens were determined by immunohistochemistry. Briefly, individual paraffin-embedded tissue sections $(4-\mu \mathrm{m})$ were deparaffinized and rehydrated. The sections were treated with $3 \% \mathrm{H}_{2} \mathrm{O}_{2}$, blocked with $5 \%$ fat-free milk, and incubated overnight with rabbit anti-STAT1 (1:500 dilution) (Bioworld Technology Inc., St. Louis Park, MN, USA). After being washed, the bound antibodies were detected with biotinylated goat-anti-rabbit IgG and visualized with horseradish peroxidase (HRP)-conjugated streptavidin and 3,3-diaminobenzidine (DAB; Sigma, St. Louis, MO, USA).

The number of positive anti-STAT1-staining hepatocytes in five areas selected randomly from a single specimen was counted, and the intensity of anti-STAT1 staining was scored by two pathologists in a blinded manner using a semi-quantitative and subjective grading system. The level of STAT1 expression was scored according to the percentage of positively stained tumor cells: $0,<5 \%$ positively stained tumor cells; $1,6-10 \%$ positively stained tumor cells; $2,11-25 \%$ positively stained tumor cells; and 3,>40\% positively stained tumor cells. The intensity of anti-STAT1 staining was scored as 0 , no staining; 1, weak staining; 2 , moderate staining; and 3, strong staining.
The staining index for each section was calculated as the score of the staining intensity multiplied by the score of the STAT1 expression levels. Cases with discrepant results were re-evaluated jointly and discussed to reach an agreement.

Cell culture. Human HCC HepG2 cells were purchased from the American Type Culture Collection (ATCC, Rockville, MD, USA). The cells were maintained in Dulbecco's modified Eagle's medium (DMEM) containing 10\% fetal calf serum (FCS), $100 \mathrm{U} / \mathrm{ml}$ of penicillin, and $100 \mu \mathrm{g} / \mathrm{ml}$ of streptomycin at $37^{\circ} \mathrm{C}$ in a $5 \% \mathrm{CO}_{2}$-humidified incubator.

Cell transfection. The sense and antisense sequences of the STAT1-specific and control siRNAs were synthesized by Shanghai GenePharma, Shanghai, China and are presented in Table I. HepG 2 cells (1x10\% /well) were cultured in 6-well plates overnight and transfected in duplicate with $1.3 \mu \mathrm{g}$ control or each type of STAT1-specific siRNA using X-tremeGENE siRNA transfection reagent, according to the manufacturer's instructions (Roche, Mannheim, Germany). The plasmids of pcDNA3.1 (EV) and pcDNA3.1-STAT1 were obtained from GenePharma. HepG2 cells (1x10\%/well) were transfected in duplicate with $2 \mu \mathrm{g}$ pcDNA3.1 (EV) and pcDNA3.1-STAT1 using X-tremeGENE DNA transfection reagents. Forty-eight hours after transfection, the cells were harvested and used for the following experiments.

Quantitative real-time reverse transcription-polymerase chain reaction ( $q R T-P C R$ ). Total RNA was extracted from each cell group using an RNA extraction kit (RNAiso Plus), according to the manufacturer's instructions (Takara, Tokyo, Japan). The purified RNA was reversely transcribed into cDNA. The relative levels of target gene mRNA transcripts to the control GAPDH were determined by qRT-PCR on an ABI PRISM 7300 system (ABI, USA) using the SYBR ${ }^{\circledR}$ Premix Ex $\mathrm{Taq}^{\mathrm{TM}}$ II (Takara) and specific primers. The sequences of primers were forward, 5'-GCAGGTTCACCAGCTTTATGA-3' and reverse, 5'-TGAAGATTACGCTTGCTTTTCCT-3' for STAT1 (225 bp); forward, 5'-GTTATAAGGGAGACGGG GAG-3' and reverse, 5'-TGCTCTGCTTCTTACCGCTC-3' for cyclin E (205 bp); forward, 5'-CAGATCCCTTAGTTTTG GGTGC-3' and reverse, 5'-GCCTGGAGAGACCTAGAC CA-3' for p53 (132 bp); forward, 5'-TCACTGCCACCCAGA AGACT-3' and reverse 5'-AAGGCCATGCCAGTGAGC-3' for 


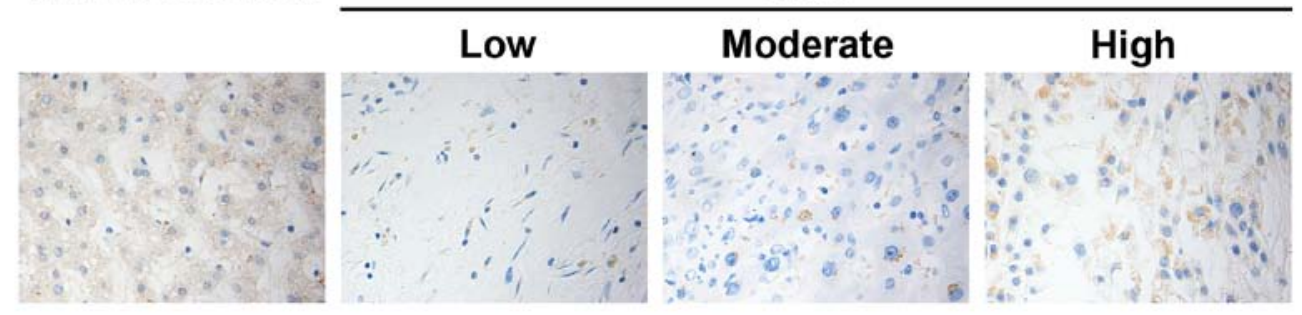

Figure 1. Immunohistochemical analysis of STAT1 expression in non-HCC liver and HCC tissues. Non-tumor liver tissues and HCC tissues at different degrees of differentiation were subjected to immunohistochemstry using a specific anti-STAT1 antibody. Data shown are representative images (magnification, $\mathrm{x} 400$ ) from each group of tissue samples. The cells with yellow-brown staining are immunopositive for STAT1.

GAPDH (157 bp). The amplifications were performed at $95^{\circ} \mathrm{C}$ for $30 \mathrm{sec}$ and subjected to 40 cycles of $95^{\circ} \mathrm{C}$ for $5 \mathrm{sec}$, and $56^{\circ} \mathrm{C}$ for $35 \mathrm{sec}$, followed by a cycle of $95^{\circ} \mathrm{C}$ for $15 \mathrm{sec}, 60^{\circ} \mathrm{C}$ for $1 \mathrm{~min}$ and $95^{\circ} \mathrm{C}$ for $15 \mathrm{sec}$. The relative expression level of each gene in individual samples was calculated using the $2^{-\Delta \Delta C T}$ method.

Western blotting. Each type of cell was lysed using RIPA lysis buffer [50 mM Tris- $\mathrm{HCl}$ (pH 7.4), $150 \mathrm{mM} \mathrm{NaCl,} 1 \%$ NP-40, $0.1 \% \mathrm{SDS}]$. After quantification of the protein concentration using the Bradford assay, cell lysates (20 $\mu \mathrm{g} / \mathrm{lane})$ were separated by $10 \%$ sodium dodecyl sulfate-polyacrylamide gel electrophoresis (SDS-PAGE), and transferred to polyvinylidene difluoride (PVDF) membranes (Millipore, Billerica, MA, USA). The membranes were blocked with $5 \%$ non-fat dry milk in Tris-buffered saline plus Tween-20 (TBS-T; 0.1\% Tween-20) and probed at $4^{\circ} \mathrm{C}$ overnight with anti-STAT1 (1:2,000 dilution) (Bioworld Technology), anti-p-STAT1 (1:2,000 dilution; Bioworld Technology), anti-p53 (1:400 dilution), anti-cyclin E (1:400 dilution) (Beijing Biosynthesis Biotechnology Co., Ltd., Beijing, China), and anti- $\beta$-actin or control IgG (1:2,000 dilution) (Wuhan Amyjet Scientific Co., Ltd., Wuhan, China). After being washed with TBS-T, the bound antibodies were detected with HRP-conjugated specific secondary antibodies for $1 \mathrm{~h}$ at room temperature and visualized using the enhanced chemiluminescent (ECL) kit (Haigene, Harbin, China). The relative levels of each target protein to the control $\beta$-actin were determined using a UVP bioimaging system and LabWorks 4.6 software (UVP, Upland, CA, USA).

MTT assay. The impact of STAT1 on HCC cell proliferation was evaluated by MTT (3-(4,5-dimethylthiazol-2-yl)-2,5-diphenyltetrazolium bromide) assay. HCC cells $\left(2 \times 10^{4} /\right.$ well $)$ were cultured on 96-well plates overnight and were transfected in triplicate with $0.1 \mu \mathrm{g} /$ well plasmid vectors or siRNAs for $48 \mathrm{~h}$. Subsequently, the cells were exposed to $100 \mu \mathrm{l}$ of MTT solution (R\&D Systems, Minneapolis, MN, USA) and continually cultured for $4 \mathrm{~h}$. The formed formazan was dissolved with MTT solubilization buffer and measured for the absorbance at $570 \mathrm{~nm}$ on an ELISA plate reader.

Flow cytometric analysis. After transfection for $48 \mathrm{~h}$, the cells were harvested and stained with FITC-Annexin V and propidium iodide (PI) (Invitrogen, Grand Island, NY, USA) in the dark for $20 \mathrm{~min}$ and washed with staining buffer. The
Table II. STAT1 expression in non-HCC liver and HCC tissues.

\begin{tabular}{lrrrrrrrr}
\hline & \multicolumn{6}{c}{ STAT1 expression } & \\
\cline { 3 - 6 } & $\mathrm{N}$ & 0 & 1 & 2 & 3 & 4 & P-value \\
\hline Non-HCC liver tissues & 12 & 1 & 1 & 1 & 2 & 7 & $<0.05$ \\
HCC tissues & 36 & 19 & 4 & 5 & 5 & 3 & \\
\hline
\end{tabular}

percentage of apoptotic cells was determined by flow cytometric analysis on a FACSAria (BD Biosciences, San Jose, CA, USA).

Statistical analysis. All results are expressed as the means \pm SEM or real case number. The difference between groups was analyzed by one-way ANOVA or the Chi-squared test, and the non-parametric data between groups were analyzed by the Mann-Whitney U test using SPSS version 17.0 software. A P-value of $<0.05$ was considered to indicate a statistically significant result.

\section{Results}

Clinical relevance of STAT1 in the development and progression of HCC. To determine the clinical relevance of STAT1 expression in the development and progression of $\mathrm{HCC}$, a total of $36 \mathrm{HCC}$ patients and 12 non-HCC patients were recruited, and their HCC and non-HCC liver tissue sections were subjected to immunohistochemical analysis (Fig. 1). As shown in Table II, the percentage of STAT1-expressing non-HCC tissues (11/12) was significantly higher than the percentage in HCC tissues $(17 / 36, \mathrm{P}<0.05)$. Quantitative analysis of STAT1 expression indicated that there was a significant difference in the relative levels of STAT1 expression between HCC and non-HCC tissues in this population $(\mathrm{P}<0.05)$. Stratification analysis revealed that the level of STAT1 expression in the HCC tissues was positively associated with the degree of HCC differentiation $(\mathrm{P}<0.05)$, but was negatively correlated with serum HBsAg, anti-HCV and $\alpha$-AFP positivity (all $\mathrm{P}<0.05$, Table III). However, the level of STAT1 expression in the HCC tissues was not associated with age, gender and tumor size in this population. These data suggest that STAT1 may be a 
Table III. Stratification analysis of the potential association between the levels of STAT1 expression and demographic and clinical characteristics of the HCC patients.

Levels of STAT1 expression

\begin{tabular}{|c|c|c|c|c|c|c|c|}
\hline Factors & $\mathrm{N}$ & 0 & 1 & 2 & 3 & 4 & $\mathrm{P}$-value \\
\hline \multicolumn{8}{|l|}{ Age (years) } \\
\hline$<50$ & 14 & 8 & 2 & 2 & 1 & 1 & $>0.05$ \\
\hline$\geq 50$ & 22 & 11 & 4 & 2 & 3 & 2 & \\
\hline \multicolumn{8}{|l|}{ Gender } \\
\hline Male & 21 & 11 & 3 & 3 & 2 & 2 & $>0.05$ \\
\hline Female & 15 & 8 & 3 & 1 & 2 & 1 & \\
\hline \multicolumn{8}{|c|}{ Tumor diameter $(\mathrm{cm})$} \\
\hline$<5$ & 13 & 7 & 1 & 1 & 3 & 1 & $>0.05$ \\
\hline$\geq 5$ & 23 & 12 & 5 & 3 & 1 & 2 & \\
\hline \multicolumn{8}{|c|}{ Differentiation } \\
\hline High & 12 & 5 & 1 & 2 & 2 & 2 & $<0.05$ \\
\hline Moderate & 12 & 6 & 2 & 1 & 2 & 1 & $<0.05$ \\
\hline Low & 12 & 8 & 3 & 1 & 0 & 0 & \\
\hline \multicolumn{8}{|l|}{ HBsAg } \\
\hline Positive & 22 & 13 & 4 & 3 & 1 & 1 & $<0.05$ \\
\hline Negative & 14 & 6 & 2 & 1 & 3 & 2 & \\
\hline \multicolumn{8}{|l|}{ Anti-HCV } \\
\hline Positive & 10 & 5 & 3 & 1 & 0 & 1 & $<0.05$ \\
\hline Negative & 26 & 14 & 3 & 3 & 4 & 2 & \\
\hline \multicolumn{8}{|l|}{ Serum AFP } \\
\hline Positive & 27 & 14 & 5 & 3 & 4 & 1 & $<0.05$ \\
\hline Negative & 9 & 5 & 1 & 1 & 0 & 2 & \\
\hline
\end{tabular}

negative regulator of the development and progression of $\mathrm{HCC}$ in humans.

Induction of STAT1 overexpression inhibits HepG2 cell proliferation and induces HepG2 cell apoptosis in vitro. To further illustrate the functional role of STAT1 in the development of HCC, we examined the effects of STAT1 overexpression by transient transfection with a recombinant plasmid encoding the STAT1 sequence (pcDNA3.1-STAT1) or control empty vector (EV) pcDNA3.1 on the proliferation and apoptosis of HepG2 cells. Forty-eight hours after transfection, the levels of STAT1 expression were determined by RT-PCR and western blot assays. The relative level of STAT1 mRNA transcripts in the pcDNA3.1-STAT1-transfected HepG2 cells was significantly higher than levels in the EV and unmanipulated HepG2 cells (data not shown). Similarly, the level of STAT1 protein in the pcDNA3.1-STAT1-transfected HepG2 cells was significantly higher than levels in the EV and unmanipulated HepG2 cells (Fig. 2A), but there was no significant difference in the levels of STAT1 expression between the EV and unmanipulated HepG2 cells. A similar pattern for phosphorylated STAT1 was detected in these groups of cells, suggesting that induction of STAT1 overexpression increased the levels of phosphorylated STAT1. Furthermore, proliferation of the pcDNA3.1-STAT1transfected HepG2 cells was significantly reduced when compared with that of the EV-transfected HepG2 cells
(Fig. 2B), but the percentage of apoptotic pcDNA3.1-STAT1transfected HepG2 cells was significantly higher (60.6 vs. $8.06 \%$ ) when compared with that of the EV-transfected HepG2 cells (Fig. 2C and D). Collectively, induction of STAT1 overexpression inhibited proliferation and promoted apoptosis in the HepG2 cells in vitro.

Knockdown of STAT1 expression promotes proliferation and inhibits apoptosis of HepG2 cells. In parallel, we tested the impact of STAT1 silencing by transfection with STAT1specific siRNA on the levels of STAT1 phosphorylation, proliferation and apoptosis in HepG2 cells. We designed and synthesized three pairs of STAT1-specific siRNAs. Following transfection, we found that all of the tested siRNAs effectively reduced the levels of STAT1 expression, and transfection with siRNA3 resulted in inhibition of STAT1 expression by $\sim 65 \%$ (Fig. 3A). Notably, the relative level of phosphorylated STAT1 to total STAT1 in the STAT1-knockdown (KD) HepG2 cells was higher than those levels in the EV-transfected and unmanipulated HepG2 cells. While the proliferation of STAT1-KD HepG2 cells was significantly increased when compared with that of the unmanipulated HepG2 cells (Fig. 3B), the percentage of apoptotic STAT1-KD HepG2 cells was significantly less than that of the unmanipulated HepG2 cells ( 8.43 vs. $13.1 \%$, Fig. 3C and D). Therefore, knockdown of STAT1 expression 

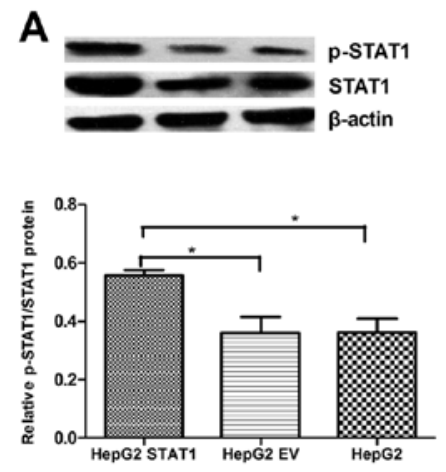

B

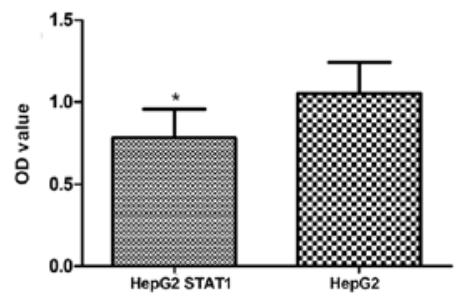

C

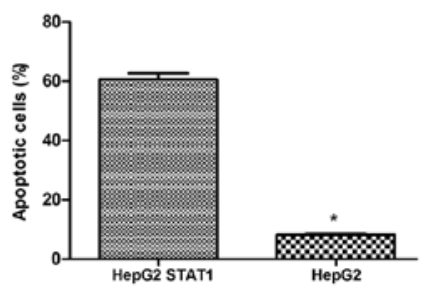

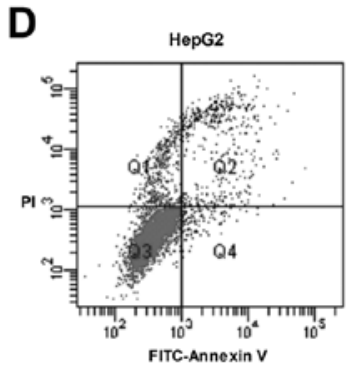

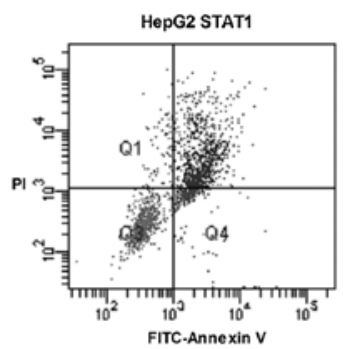

Figure 2. Induction of STAT1 overexpression inhibits proliferation and enhances apoptosis in HepG2 cells. HepG2 cells were transfected with pcDNA3.1STAT1 or empty vector pcDNA3.1 (EV) for $48 \mathrm{~h}$, and the relative levels of STAT1 mRNA transcripts and protein were characterized by RT-PCR and western blot assays. The proliferation and apoptosis of the transfected and unmanipulated HepG2 cells were determined by MTT and flow cytometric analysis, respectively. Data are representative images or expressed as the means \pm SEM of individual groups of cells from three separate experiments. (A) Western blot analysis. (B) HepG2 cell proliferation. (C and D) HepG2 cell apoptosis. A similar pattern of STAT1 mRNA transcripts was detected in the different groups of cells (data not shown). ${ }^{\prime} \mathrm{P}<0.05$, as determined by the Student's t-test.
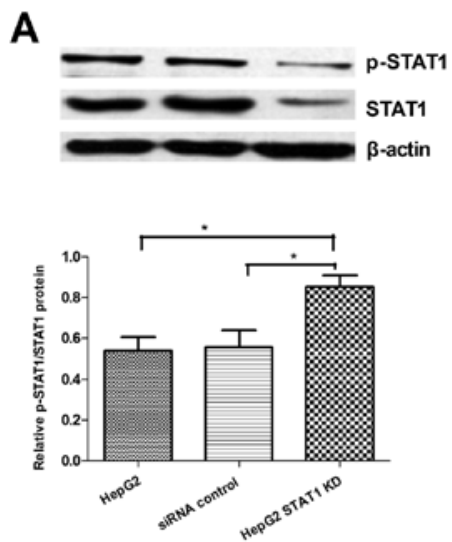

D

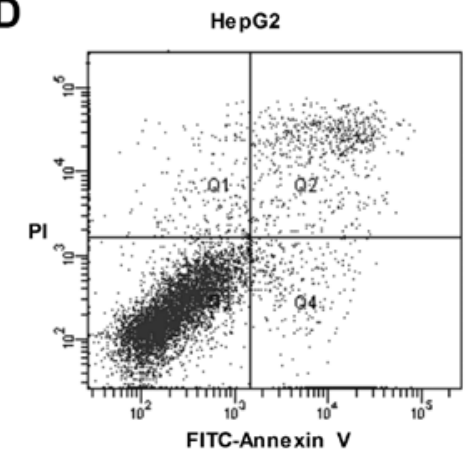

B

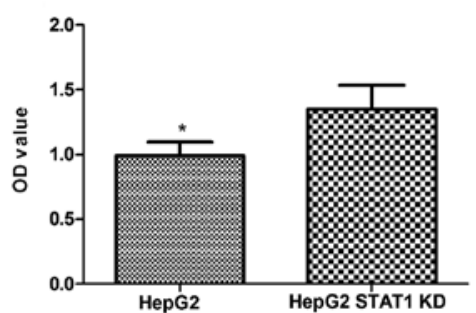

C

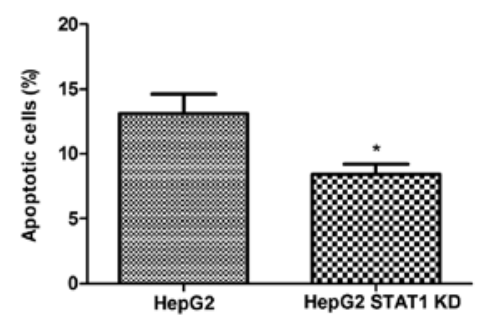

HepG2 STAT1 KD

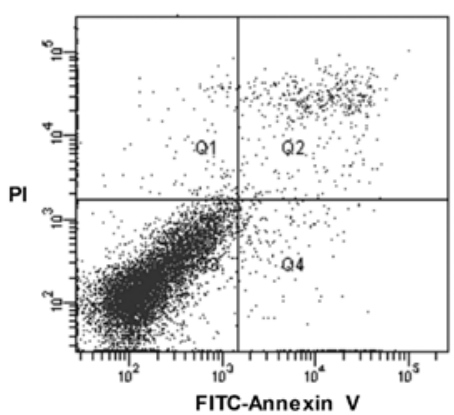

Figure 3. Knockdown of STAT1 promotes proliferation and inhibits apoptosis in HepG2 cells. HepG2 cells were transfected with three different STAT1specific siRNAs or control siRNA for $48 \mathrm{~h}$, and the relative levels of STAT1 mRNA transcripts and protein were determined by RT-PCR and western blot assays. The inhibitory effects of transfection with STAT1-specific siRNA1 and siRNA2 were less when compared with the effect of transfection with STAT1specific siRNA3 (data not shown). Subsequently, HepG2 cells were transfected with STAT1-specific siRNA3 or control siRNA for $48 \mathrm{~h}$. The proliferation and apoptosis of the different groups of HepG2 cells were determined by MTT and flow cytometric analysis. Data are representative images or expressed as the means \pm SEM of individual groups of cells from 3 separate experiments. (A) Western blot analysis. (B) HepG2 cell proliferation. (C and D) HepG2 cell apoptosis. A similar pattern of STAT1 mRNA transcripts was detected in the different groups of cells (data not shown). "P $<0.05$, as determined by the Student's t-test. 
A
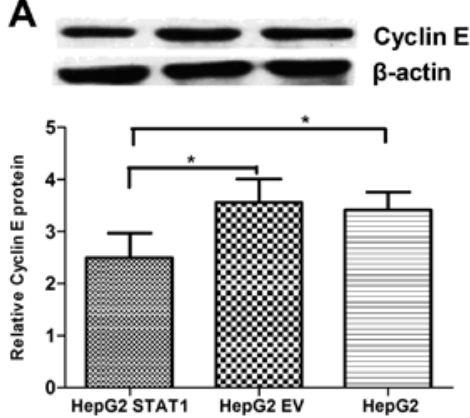

C
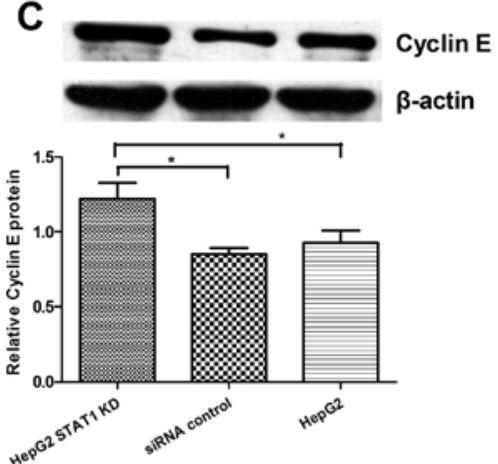

E
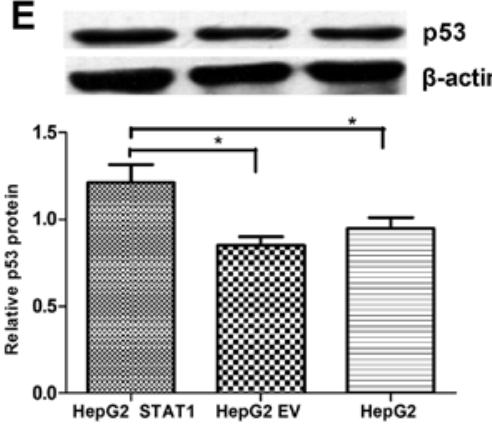

G
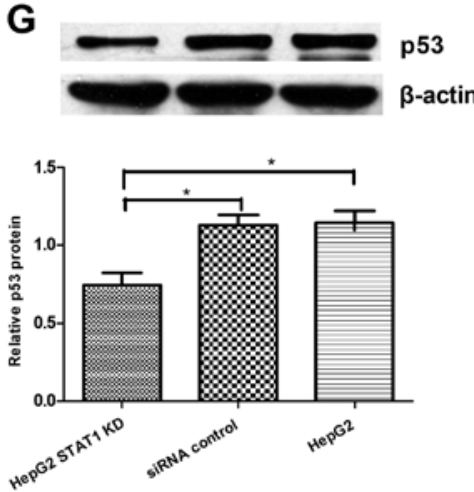

B

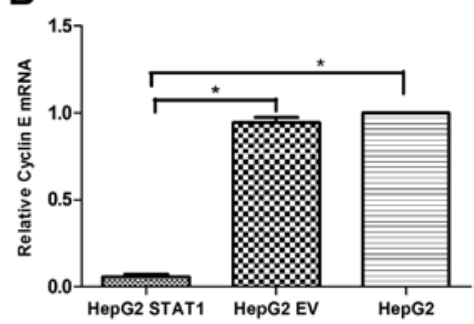

D

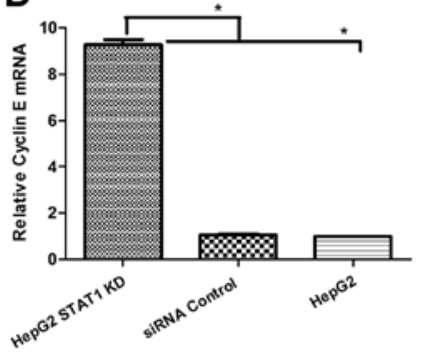

F

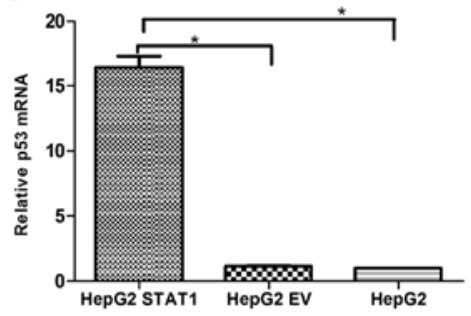

H

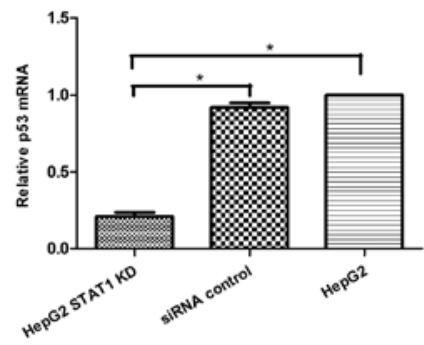

Figure 4. Modulation of STAT1 expression alters the relative levels of cyclin E and p53 expression in HepG2 cells. HepG2 cells were transfected with pcDNA3.1-STAT1, EV, control siRNA or STAT1-specific siRNA for $48 \mathrm{~h}$. The relative levels of cyclin E and p53 mRNA transcripts and proteins in the different groups of cells were determined by western blot assays (A, C, E and G) and RT-PCR (B, D, F and H), respectively. Data are representative images or expressed as the means \pm SEM of individual groups of cells from 3 separate experiments. "P<0.05, as determined by the Student's t-test.

promoted proliferation and inhibited apoptosis in the HepG2 cells in vitro.

Modulation of STAT1 expression alters the levels of cyclin $E$ and p53 expression in HepG2 cells. Cyclin E and p53 are crucial regulators of cell proliferation and apoptosis. To understand the mechanisms underlying the action of STAT1, we examined the relative levels of cyclin E and p53 expression in the different groups of HepG2 cells. The relative level of cyclin E expression in the pcDNA3.1-STAT1-transfected HepG2 cells was significantly lower than levels in the EV-transfected and unmanipulated HepG2 cells (Fig. 4A and B). In contrast, the relative level of cyclin E expression in the STAT1-KD HepG2 cells was significantly higher than levels in the control siRNA-transfected and unmanipulated HepG2 cells (Fig. 3C and D). In addition, the relative level of p53 expression in the 
pcDNA3.1-STAT1-transfected HepG2 cells was significantly higher than those in the EV-transfected and unmanipulated HepG2 cells, while the relative level of p53 expression in the STAT1-KD HepG2 cells was significantly lower than levels in the control siRNA-transfected and unmanipulated HepG2 cells (Fig. 3E-H). Thus, induction of STAT1 overexpression downregulated cyclin E expression, but upregulated p53 expression in HepG2 cells. In contrast, knockdown of STAT1 expression increased the level of cyclin E, but decreased the level of p53 expression in HepG2 cells.

\section{Discussion}

The STAT family members were originally identified as cytokine-related signal events and have emerged as promising molecular targets for cancer therapy (11). Among these proteins, STAT1 plays a critical role in IFN- $\gamma$-dependent signaling in the regulation of diverse biological actions, including antiviral defense $(12,13)$, induction of cell death and growth arrest $(14,15)$.

In the present study, we found significantly lower levels of STAT1 expression in HCC tissues, consistent with a previous study that demonstrated significantly lower levels of STAT1 expression in squamous cell carcinoma (16). Furthermore, we found that the level of STAT1 expression in HCC tissues was positively correlated with the degree of differentiation of HCC and was associated negatively with serum $\mathrm{HBsAg}$, anti-HCV and $\alpha$-AFP positivity in HCC patients. It is well known that $\mathrm{HBV}$ or HCV infection is a risk factor for the development of $\mathrm{HCC}$ and $\alpha$-AFP positivity occurs at a high frequency in HCC patients, contributing to the progression of HCC. Hence, our novel data suggest that STAT1 may be a negative regulator of the development and progression of HCC. Given that the histological degree of HCC is associated with the prognosis of HCC, further investigation to determine whether the level of STAT1 expression in HCC tissues could serve as a biomarker for evaluating the prognosis of HCC in Chinese patients is warranted. However, it is notable that ubiquitous activation of Ras and Jak/Stat pathways is detected in human HCC tissues (17). Such a discrepancy may be due to the different genetic backgrounds and variable pathologic factors that contribute to the development of HCC. While chronic viral hepatitis is the major factor for the development of HCC in the Chinese population, hyperlipidemia-related and alcoholic liver diseases are crucial for the development of HCC in Western countries. Indeed, the levels of STAT1 expression vary in different types of cancers, and even in the same type of cancer with different genetic backgrounds and in patients from different geographic regions (16). We are interested in further investigating how these factors modulate the STAT1 expression and activation, contributing to the development and progression of HCC.

To understand the role of STAT1 in the development and progression of $\mathrm{HCC}$, we evaluated the impact of altered levels of STAT1 expression on the proliferation and apoptosis of HepG2 cells. Our results support the notion that STAT1 may be a tumor suppressor. We found that induction of STAT1 overexpression inhibited proliferation, but enhanced apoptosis of HepG2 cells. Similarly, knockdown of STAT1 expression enhanced proliferation, but inhibited apoptosis of HepG2 cells. These two independent lines of evidence clearly indicate that
STAT1 acts as a suppressor of HCC cell proliferation, which is consistent with a previous study of human head and neck squamous cell carcinoma (16).

Several molecules have been implicated in STAT1regulated cell survival, proliferation and apoptosis. These include caspase-2, -3 and -7, Bcl-2, IRF1 and p21/Waf1 (18-20). Cyclin $\mathrm{E}$ is associated with CDK2 and is crucial for regulating the G1/S phase transition of the cell cycle (21). It has been reported that IFN- $\gamma$ induces G0/G1 phase arrest in human melanoma cells by downregulating cyclin $\mathrm{E}$ and cyclin-dependent kinase expression (22). We found that induction of STAT1 overexpression in HepG2 cells significantly reduced the levels of cyclin E expression, while knockdown of STAT1 expression dramatically upregulated the levels of cyclin E expression in HepG2 cells. These data suggest that STAT1 inhibits cyclin E expression and induces cell cycle arrest in HCC cells. In addition, a previous study indicated that loss of p53 expression or the presence of abnormal forms of the protein is frequently detected in HCC cell lines, suggesting that alterations in p53 expression may be an important event in the transformation of hepatocytes to the malignant phenotype (23). In the present study, we found that induction of STAT1 overexpression increased the levels of p53 expression, while knockdown of STAT1 expression decreased the levels of p53 expression in HepG2 cells. Indeed, a previous study demonstrated that p53 is an important negative regulator of the cell cycle in HCC cells (24). It is possible that STAT1 may regulate the p53 expression and in turn inhibit cyclin E expression, leading to cell cycle arrest, growth inhibition, and apoptosis of HCC cells.

In summary, our data revealed a significantly lower level of STAT1 expression in HCC tissues, which was correlated positively with the degree of differentiation of HCC, but was negatively associated with the positivity of $\mathrm{HBV}, \mathrm{HCV}$ infection, and AFP in Chinese HCC patients. Furthermore, our data also suggest that STAT1 may function as a suppressor of HCC cell proliferation and a regulator of HCC cell apoptosis, which is modulated by an increased level of p53 expression and a decreased level of cyclin E expression in HepG2 cells. Hence, our findings highlight the importance of STAT1 in regulating the development and progression of HCC. Conceivably, our findings may provide a basis for the design of new therapies for the intervention of HCC in the clinic. We recognize that our study had limitations due to its small sample size and the lack of longitudinal studies. Therefore, further longitudinal investigation in a larger population is warranted.

\section{Acknowledgements}

We thank all members of the Laboratory of the General Surgery Department (The First Hospital of Jilin University) and Haihe Wang (Harbin Medical University) for their insights and technical support. We thank Medjaden Bioscience Limited for assisting in the preparation of this manuscript.

\section{References}

1. Farazi PA and DePinho RA: Hepatocellular carcinoma pathogenesis: from genes to environment. Nat Rev Cancer 6: 674-687, 2006.

2. Llovet JM and Bruix J: Molecular targeted therapies in hepatocellular carcinoma. Hepatology 48: 1312-1327, 2008. 
3. Lodige I, Marg A, Wiesner B, Malecova B, Oelgeschlager T and Vinkemeier U: Nuclear export determines the cytokine sensitivity of STAT transcription factors. J Biol Chem 280: 43087-43099, 2005.

4. Mowen K and David M: Regulation of STAT1 nuclear export by Jak1. Mol Cell Biol 20: 7273-7281, 2000.

5. Khodarev NN, Minn AJ, Efimova EV, et al: Signal transducer and activator of transcription 1 regulates both cytotoxic and prosurvival functions in tumor cells. Cancer Res 67: 9214-9220, 2007.

6. Bromberg JF, Horvath CM, Wen Z, Schreiber RD and Darnell JE Jr: Transcriptionally active Stat1 is required for the antiproliferative effects of both interferon $\alpha$ and interferon $\gamma$. Proc Natl Acad Sci USA 93: 7673-7678, 1996.

7. Shankaran V, Ikeda H, Bruce AT, White JM, Swanson PE, Old LJ and Schreiber RD: IFN $\gamma$ and lymphocytes prevent primary tumour development and shape tumour immunogenicity. Nature 410: 1107-1111, 2001.

8. Osna NA, Clemens DL and Donohue TM Jr: Ethanol metabolism alters interferon gamma signaling in recombinant HepG2 cells. Hepatology 42: 1109-1117, 2005.

9. Lin W, Choe WH, Hiasa Y, Kamegaya Y, Blackard JT, Schmidt EV and Chung RT: Hepatitis C virus expression suppresses interferon signaling by degrading STAT1. Gastroenterology 128 : 1034-1041, 2005

10. Bromberg J and Darnell JE Jr: The role of STATs in transcriptional control and their impact on cellular function. Oncogene 19: 2468-2473, 2000

11. Yu H and Jove R: The STATs of cancer - new molecular targets come of age. Nat Rev Cancer 4: 97-105, 2004.

12. Levy DE and Darnell JE Jr: Stats: transcriptional control and biological impact. Nat Rev Mol Cell Biol 3: 651-662, 2002.

13. Samuel CE: Antiviral actions of interferons. Clin Microbiol Rev 14: 778-809, 2001.

14. Balasubramanian A, Ganju RK and Groopman JE: Signal transducer and activator of transcription factor 1 mediates apoptosis induced by hepatitis $C$ virus and HIV envelope proteins in hepatocytes. J Infect Dis 194: 670-681, 2006.
15. Chawla-Sarkar M, Lindner DJ, Liu YF, Williams BR, Sen GC, Silverman RH and Borden EC: Apoptosis and interferons: role of interferon-stimulated genes as mediators of apoptosis. Apoptosis 8: 237-249, 2003

16. Xi S, Dyer KF, Kimak M, et al: Decreased STAT1 expression by promoter methylation in squamous cell carcinogenesis. J Nat Cancer Inst 98: 181-189, 2006.

17. Calvisi DF, Ladu S, Gorden A, et al: Ubiquitous activation of Ras and Jak/Stat pathways in human HCC. Gastroenterology 130: 1117-1128, 2006.

18. Lee CK, Smith E, Gimeno R, Gertner R and Levy DE: STAT1 affects lymphocyte survival and proliferation partially independent of its role downstream of IFN- $\gamma$. J Immunol 164: 1286-1292, 2000.

19. Nguyen H, Lin R and Hiscott J: Activation of multiple growth regulatory genes following inducible expression of IRF-1 or IRF/RelA fusion proteins. Oncogene 15: 1425-1435, 1997.

20. Sironi JJ and Ouchi T: STAT1-induced apoptosis is mediated by caspases 2, 3, and 7. J Biol Chem 279: 4066-4074, 2004.

21. Knoblich JA, Sauer K, Jones L, Richardson H, Saint R and Lehner CF: Cyclin E controls S phase progression and its downregulation during Drosophila embryogenesis is required for the arrest of cell proliferation. Cell 77: 107-120, 1994.

22. Kortylewski M, Komyod W, Kauffmann ME, Bosserhoff A, Heinrich PC and Behrmann I: Interferon- $\gamma$-mediated growth regulation of melanoma cells: involvement of STAT1-dependent and STAT1-independent signals. J Invest Dermatol 122: 414-422, 2004.

23. Bressac B, Galvin KM, Liang TJ, Isselbacher KJ, Wands JR and Ozturk M: Abnormal structure and expression of p53 gene in human hepatocellular carcinoma. Proc Natl Acad Sci USA 87: 1973-1977, 1990

24. Sheahan S, Bellamy CO, Dunbar DR, Harrison DJ and Prost S: Deficiency of G1 regulators P53, P21 $1^{\text {Cipl }}$ and/or pRb decreases hepatocyte sensitivity to TGF $\beta$ cell cycle arrest. BMC Cancer 7: 215, 2007. 